Notre Dame Journal of Formal Logic Volume XIV, Number 2, April 1973 NDJFAM

\title{
ON HOW BEST TO MAKE SENSE OF LEŚNIEWSKI'S ONTOLOGY
}

\author{
PAUL T. SAGAL
}

Familiarity breeds contempt; on the other hand it can be very comforting. Philosophers find familiar logical systems very comforting. On the whole they prefer the logic they learned on their mother's knee or in graduate school. When confronted with an unfamiliar system they either resist it or twist and turn to put the unfamiliar in a familiar frame. A. N. Prior, in his essay Existence in Leśniewski and in Russell ${ }^{1}$ does a lot of twisting and turning. Prior centers his discussion upon Theorem 24.52 of Russell and Whiteheads's Principia Mathematica. This theorem asserts that there exists at least one individual. But where does a logical system come off telling us that something exists? Leśniewski's ontology contains no such thesis. Prior's essay investigates how ontology could get away with this when Russell considered 24.52 a necessary evil. This investigation leads Prior to make some general claims about Leśniewski's ontology, and to present its basic ideas in what Prior considers a less puzzling way than is customary. Prior's thesis is "that ontology is just a broadly Russellian theory of classes deprived of any variables of Russell's lowest logical type." (150) If we consider lowest type variables to range over individuals then we are left with a no individual theory. The only logical truths which remain would be those not involving individuals. According to Prior, the above characterization captures the essence of ontology. To give the reader who is completely unfamiliar with ontology enough information to appreciate the following discussion, I will make a few observations about ontology. Ontology is constructed upon a basis provided by the propositional calculus (P.C.), Leśniewski's own version of the P.C. (called protothetic) was an extended version of familiar systems. For example, quantification (of the substitutional variety) ${ }^{2}$ was already introduced at that

1. A. N. Prior, "Existence in Leśniewski and Russell," in Formal Systems and Recursive Functions, ed. by Crosley and Dummett, North Holland (1963). (Page references in paper will be to Prior's essay.)

2. For substitutional quantification see W. V. Quine, 'Existence and Quantification' in $[5]$. 
level. Ontology introduces a single new logical constant the $\epsilon$ and with it two new grammatical or syntactical categories. The $\epsilon$ is a sentence forming functor, forming sentences from names. (The new categories are (1) names and (2) sentence forming functors with names). The $\epsilon$ is introduced by a single axiom. The semantics for a sentence of the form $a \epsilon b$ is (i) ' $a$ ' names at least one thing, (ii) ' $a$ ' names at most one thing, (iii) anything ' $a$ ' names is also named by ' $b$.' The axiom itself may be viewed as presenting truth conditions for $\epsilon$ sentences. It goes like this $[a, b][(a \epsilon b) \equiv$ $(\mathrm{E} c)(c \in a) .(c)(d)(c \epsilon a . d \epsilon a \rightarrow c \epsilon d) .(\mathrm{c})(c \epsilon a \rightarrow c \epsilon d)]$. With the ontological epsilon we can introduce an exists (singular existence) functor as follows: (a) $a$ exists $\equiv(\mathrm{E} b)(b \in a)$. The reader is reminded that no exists (or $\epsilon$ ) statement (a statement with $\epsilon$ as the main functor) is a thesis of ontology. Quantification is totally divorced from matters of existence. Note too that the ontological $\epsilon$ differs from Russell's set theoretical epsilon in that it connects homogeneous terms (terms belonging to the same grammatical category).

It is no easy matter to cut away the individual variables from Russell's theory. As Prior admits, "the formal development of a Russellian class theory without variables of the lowest type presents, however, some very taxing problems."' (150) Why is this? Because on Russell's account sets are ultimately composed of individuals (and functions of individuals). Wipe out the individuals and you no longer have a coherent picture of Russellian sets. How can there be sets of individuals without individuals? "Leśniewski meets this difficulty by introducing an undefined constant expressing a relation between classes-it can be, but it does not need to be the functor ' $\epsilon$ ' previously mentioned. This functor, as I have also previously said, has arguments of the same logical type, so that what it expresses is not Russellian class-membership. It expresses rather the inclusion of a unit class in another class." (151) A word of caution is in order here. Prior's explicit talk of functors expressing relations and implicit talk of variables ranging over classes is quite out of spirit with the sharp separation ontology makes between questions of grammar and questions of existence. (Prior however comes back to this point.) Functors need not designate or express functions. Names need not name anything. Variables need not have a range. Put another way, Leśniewski's ontology attributes no special categorematic status to any grammatical category. Ontology treats all categories of expressions syncategorematically. ${ }^{3}$

Principia Mathematica (PM) assumes that its lowest type variables have individual names for substituends. Such variables range over individuals. Existence assumptions are involved in the basic quantificational logic of PM. The universe cannot be empty because quantificational logic will not permit it. "One way of purging logic of this assumption would be to conceive quantification theory as being concerned simply with the

3. My forthcoming Languages and Ontology will contain further discussion of these matters. 
application of quantifiers to functors with their arguments without regard to what parts of speech these functors and arguments are. The form of quantification theory would in fact be unchanged if we interpreted Russell's lowest type variables as standing for Lesniewskian 'names', that is to say class names, and his predicates for functors forming sentences out of these." (153) To this point, what has happened to the Russellian theory of classes in the process of bringing it into line with Leśniewski's ontology? (1) It has been purged of the lowest level of variables (individual variables). (2) The notion 'variable' itself has been re-interpreted so it could be incorporated in a non-Russellian (substitutional) theory of quantification. (3) The $\epsilon$ of set membership has become the $\epsilon$ of unit class inclusion. (The Russellian view of sets as being composed of individuals has also bitten the dust.) Even at this juncture we may well wonder how seriously to take Prior's enunciation that ontology is just a broadly Russellian theory of classes. Perhaps 'broadly' is to be taken extremely broadly.

Towards the end of his essay, Prior admits that ontology-the broadly Russellian theory of classes-is not committed to the existence of classes at all. If a proper verb or predicate is a functor which forms sentences from individual names, then ontological functors are not proper verbs at all. They form sentences out of nouns of any sort (shared, unshared, or empty).

And the functors which join the $a$ 's and $b$ 's in ontology, and the $\alpha$ 's and $\beta$ 's in class theory, are not, properly speaking, predicates; they are functors like "every __ is a __," "The ___ is a __, there is no such thing as a _... . It is no doubt convenient to use forms like "The class of $a$ 's is an empty class", "The class of $a$ 's is a member of the class of pairs", and so on, and Leśniewski introduces a higher order " $\varepsilon$ ", which is so defined that " $f \varepsilon g$ " may be read as "The unit-class-of-classes $f$ is included in the class-of-classes $g . "$ But these are no more than convenient locutions; "The class of a's is an empty class", for example, means no more than "there is no such thing as an a" (my emphasis) from which the suggestion of naming an abstract object, the class of $a$ 's has been removed." (153-154).

Prior fails to appreciate the philosophical advantages of multiplying linguistic forms. If one does not distinguish questions of meaning from questions of reference (designation) then one sees this multiplication as a convenience and nothing more. The multiplication is easily eliminable. The higher order forms like $f \epsilon g$ introduce no new entities (they simply introduce new parts of speech), they are ontologically impotent. We do not really need them. But these observations do not touch upon questions of meaning proper (questions of meaning in Quine's sense). ${ }^{4}$ Ontology, of course, does not provide us with a theory of meaning. However, and this is important, it leaves us great flexibility in assigning meanings, in interpreting sentences, through its multiplicity of linguistic forms. Languages

4. See W. V. Quine, 'On What There Is' in [4]. 
which do not multiply forms of expression must treat the same expression as having different meanings in different contexts if they are to retain what might be termed semantic flexibility.

So contra Prior, we are in no way compelled to treat expressions like "the class of $a$ 's is an empty class" and "there is no such thing as an $a$ " as alike in meaning. The expressions are equivalent in ontology, but such equivalence need not involve synonomy, or great likeness in meaning. The importance of having many linguistic forms, and many parts of speech available has proved extremely useful in interpreting and reconstructing difficult positions from the philosophical past. ${ }^{5}$ By way of conclusion, I leave it to the reader to decide whether Leśniewski's ontology is best presented as a broadly Russellian theory of classes.

\section{SELECTED BIBLIOGRAPHY}

[1] Henry, D. P., The De Grammatico of St. Anselm, University of Notre Dame Press (1964).

[2] Kearns, J. T., "The contribution of Leśniewski," Notre Dame Journal of Formal Logic, vol. VIII (1967), pp. 61-93.

[3] Lejewski, C., “On Leśniew'ski's Ontology,' Ratio, vol. 1 (1958), pp. 150-176.

[4] Quine, W. V., From a Logical Point of View, Harper Torchbooks (1961).

[5] Quine, W. V., Ontological Relativity and Other Essays, Columbia University Press (1969).

Boston University

Boston, Massachusetts

5. See D. P. Henry, [1], and my own Languages and Ontology (forthcoming). 